O. Lysenko, A. Mamalis, V. Andruschenko and E. Mitskevich

\title{
Surface nanomachining using scanning tunnelling microscopy with a diamond tip
}

\begin{abstract}
The results of the utilization of a scanning tunnelling microscope (STM) for surface nano-machining are presented. The instrument's peculiarity is the electromagnetic probe-tosurface load measuring system. Purposely synthesized boron-doped single diamond crystals were used as tips. Silicon surface scratching, mechanical removal of fragments of gold film and gold surface cleanup are demonstrated. Surface cleanup quality was estimated through measurements of the voltage-current characteristics of tunnelling. Prospects for the exploitation of the diamond tip-equipped STMs for nanoelectronic component fabrication are discussed.
\end{abstract}

Nanotechnology Perceptions 6 (2010) 41-49

Nonsubscribers: purchase individual article 